\title{
Online Hand Written Character Recognition
}

\author{
Farha Mehdi ${ }^{1}$, Srinivasa $\mathrm{G}^{2}$, Ashwini A.J ${ }^{3}$, Hemanth Krishna H.K ${ }^{4}$ \\ 1.Asst.prof, Dept of ISE, APSCE, Bangalore, \\ 2.Asst.prof, Dept of CSE , GSSIT, Bangalore, \\ 3.Asst.prof,Dept of CSE,GSSIT, Bangalore, \\ 4. Asst.prof,Dept of CSE,AIT,Bangalore,
}

\begin{abstract}
Handwriting processing is a domain in great expansion. The interest devoted to this field is not explained only by the exciting challenges involved, but also the huge benefits that a system, designed in the context of a commercial application, could bring Two classes of recognition systems are usually distinguished: online systems for which handwriting data are captured during the writing process, which makes available the information on the ordering of the strokes, and offline systems for which recognition takes place on a static image captured once the writing process is over. The field of personal computing has begun to make a transition from the desktop to handheld devices, thereby requiring input paradigms that are more suited for single hand entry than a keyboard. Online handwriting recognition allows for such input modalities. On-line handwritten scripts are usually dealt with pen tip traces from pen-down to pen-up positions.
\end{abstract}

Keywords: Training, pre-processing, recognition, strokes, segmentation.

\section{Introduction}

During the past thirty years, substantial research efforts have been devoted to character recognition that is used to translate human readable characters to machine-readable codes. Immense effort has been made on character recognition, as it provides a solution for processing large volumes of data automatically in a large variety of scientific and business applications. The main problem encountered when dealing with handwritten English characters is that characters written by different persons representing the same character are not identical but can vary in both size and shape. The fast variation in personal writing styles and differences in one person's writing style depending on the context is another problem encountered when trying to recognize English handwritten characters. In addition, the mood of the writer and the writing situation can have an effect on writing styles.

Handwriting recognition principally entails optical character recognition. However, a complete handwriting recognition system also handles formatting, performs correct segmentation into characters and finds the most plausible words. The main problem encountered when dealing with handwritten characters is that characters written by different persons representing the same character are not identical but can vary in both size and shape. The fast variation in personal writing styles and differences in one person's writing style depending on the context is another problem encountered when trying to recognize handwritten characters. In addition, the mood of the writer and the writing situation can have an effect on writing styles.

Handwritten text recognition systems have achieved recently significant progress, due to developments in segmentation, recognition and language models. Those systems are less powerful when the languages to be recognized have a two dimensional (2D) layout. This is the case for mathematical expressions, schemas, diagrams etc. In this case, it yields to solve the same problems of segmentations, recognition and interpretation but in a 2D context. Resolving bi-dimensional languages recognition problem implies being capable of solving three sub problems: segmentation, symbol recognition and interpretation.

\subsection{Objectives}

The main objective is to recognize online handwritten documents, which includes characters, words, lines, paragraphs etc. There is extensive work in the field of handwriting recognition, and a number of reviews exits. Our approach is to recognize handwriting by using templates. Along with this we maintain a unique user accounts, which enables a particular user to create his/her training sets.

\section{Literature Survey}

Yoshida K. et.al [1] online handwriting recognition online handwriting recognition, where existing challenges are to cope with problems of various writing fashions, variable size for the same character, different stroke orders for the same letter, and efficient data presentation to the classifier. The similarities of distinct character shapes and the ambiguous writing further complicate the dilemma. A solitary solution of all these problems lies in the intelligent and appropriate extraction of features from the character at the time of writing. A typical handwriting recognition system focuses on only a subset of these problems. The goal of fully 
unconstrained handwriting recognition still remains a challenge due to the amount of variations found in characters. The handwriting recognition problem can be considered for various alphabets and at various levels of abstraction. The main goal of the work presented in this paper has been the development of an on-line handwriting recognition system which is able to recognize handwritten characters of several different writing styles. Due to the temporal nature of online data, this work has possible application to the domain of speech recognition as well. The work in this research aimed to investigate various features of handwritten letters, their use and discriminative power, and to find reliable feature extraction methods, in order to recognize them. A 22 feature set of sub-character primitive features has been proposed using a quite simple approach of feature extraction. This approach has succeeded in having robust pattern recognition features, while maintaining feature's domain space to a small, optimum quantity. Back propagation neural network (BPN) technique has been used as classifier and recognition rate up to $87 \%$ has been achieved even for highly distorted hand written characters.

Zafar M.F. et.al [1], proposes a online handwritten character recognition algorithm, suitable online handwritten character recognition algorithm, suitable for the home use computer. A character, written on a digitizing tablet, is expressed as a directional angle sequence. In order to recognize a quickly written multistroke character, the character pattern is converted into a single interconnected stroke pattern. The recognition is carried out using dynamic programming based pattern matching technique.

Chiang, C.C. et.al [3], proposes a handwritten character recognition system implemented by a stochastic neural net (SNN) is presented. The learning process in this neural model incorporates the stochastic information extracted from the trained characters. The merit of SNN lies in the fast learning speed obtained through an online learning algorithm, in contrast to offline learning algorithms. This SNN has been applied to design an experimental adaptive character recognition system. This system can recognize handwritten/printed English and Chinese characters. According to preliminary experimental results, the recognition rates are about 90 94\% and 80 85\% for English and Chinese characters, respectively. The system is capable of learning while recognizing new patterns.

Liangwen Zhang et.al [4], this paper proposes online handwritten English characters segmentation method based on rules. Firstly, all the local minima of the character coordinate sequences are treated as potential segmentation points; Secondly, we calculate 5 features of every potential segmentation point; finally, the potential segmentation points are modified according to the rules to get the final segmentation points. The modification contains shift, deletion and maintenance. Thus, the coordinate sequences of characters are segmented to sub-sequences of characters by the final segmentation points. The experiment conducted on UNIPEN database shows that our method is feasible and effective.

\section{Online Hand Written Character Recoginition}

Handwriting recognition (or HWR) is the ability of a computer to receive and interpret intelligible handwritten input from sources such as paper documents, photographs, touch-screens and other devices. The image of the written text may be sensed "off line" from a piece of paper by optical scanning (optical character recognition) or intelligent word recognition. Alternatively, the movements of the pen tip may be sensed "on line", for example by a pen-based computer screen surface.

\subsection{Types of Handwriting Recognition 3.1.1 Off-line}

Off-line character recognition takes a raster image from a scanner (scanned images of the paper documents), digital camera or other digital input sources. The image is binarized through threshold technique based on the color pattern (color or gray scale), so that the image pixels are either 1 or 0 .

\subsubsection{On-line}

In on-line, the current information is presented to the system and recognition (of character or word) is carried out at the same time. Basically, it accepts a string of $(x, y)$ coordinate pairs from an electronic pen touching a pressure sensitive digital tablet. Once the image is binarized in off-line case, the rest of the techniques for classification can be identical with only two basic differences. Firstly, off-line recognition happens after the writing completes and the scanned image is pre-processed. Secondly, it has no temporal information associated with the image due to which it is not known to the classifier about the way and order of writing. So, we can say, its knowledge about the character is limited. It means, off-line data only represents the final result after writing i.e.an image. Why does the global market demand follow the on-line recognition system?

On-line character recognition system, by contrast, captures the temporal or dynamic information of the writing, enhances the accuracy over off-line. Another advantage is interactivity, which means recognition errors can be corrected immediately with the series of test. Yet, adaptation of any drawings of character is also an 
advantage over off-line. When the user faces that some characters are not recognized accurately, user can alter his way of drawing until it recognizes. It means user can adapt to the machine. Conversely, recognizers are capable of adapting users' drawing, usually by storing possible samples from a large number of users for subsequent recognition. Thus, there is adaptation of user to machine and of machine to user. Electronic pen input is the direct method to compare with the both off-line and key-board entry to the system having recognition intelligence. In addition, on-line recognition improves the work-flow, the information is immediately available. However, the natural and comfortable style in writing effectively reduces difficulty at the threshold of using computers for common users.

\subsubsection{Based on Styles of Handwriting: Discrete vs. Cursive}

The difficulty of character recognition varies greatly with different writing styles. The writing style of the first three lines is commonly referred to as discrete handwriting, in which the writer is told to write each character within a bounding box or to separate each character. The writing style of the fourth lines is commonly referred to as pure cursive or connected within a word. Most people write in a mixed style, a combination of Discrete and Cursive styles, similar to the writing on the fifth line.

Both Discrete and Cursive character recognition are difficult tasks because of the great amount of variability present in the on-line handwriting signal. The variability is present both in time and signal space. Variability in time refers to variation in writing speed, while variability in signal space refers to the shape changes of the individual characters.

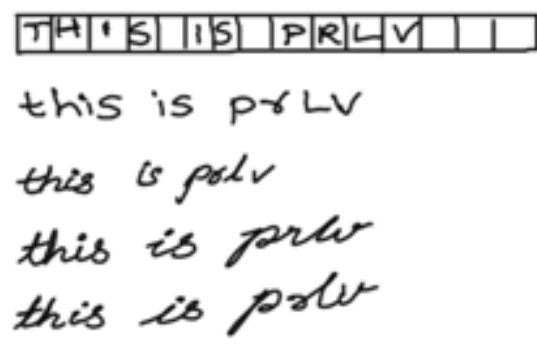

\section{Fig 2.2 Types of Handwriting}

It is rare to find two identically written characters. The difficulty of recognizing character lies in constructing accurate and robust models to accommodate the variability in time and feature space.

\subsubsection{Based on Writer Independent and Writer Dependent}

A writer independent recognition system recognizes wide ranges of possible writing styles, while a writer dependent recognition system is trained to recognize only from specific users. Therefore, a writer dependent recognition system works on data with smaller variability and therefore a chance of having higher reliability is achieved in contrast to writer independent recognition system. Writing one's style brings unevenness in writing units, which is the most difficult part. Variability in stroke numbers, their order, shape and size, tilting angle and similarity among characters from one another are found more often in writer independent recognition system. Broadly, there are two kinds of writing styles. They are hand printed and cursive character. In cursive style, strokes are deliberately linked forming one from many to draw the character, while in hand printed style possible number of strokes are used, each stroke has significant role to complete the character. In cursive style, the important information such as intersections, loops, curves, straight lines and hooks etc. are missing. Sometimes, both writing styles are mixed. Natural character contains all types of styles in writing from any of the users. Specifically, the writing is said to be natural as if users write on a piece of paper. With the introduction of portable hand held computers and computing devices such as PDAs (Personal Digital Assistant), non-keyboards and non-keypads based methods for inputting data are receiving more interest in both academic and commercial research communities. The most promising options are pen based and voice based inputs. Pen based method in inputting can be either off-line or on-line.

\section{Proposed Methodologies With Architecture Design}

Three steps are involved in the recognition of a character in this project. These methods scan the segmented character of the user and matches with the stored training set or templates. Each of the methods increases the probabilities of the templates which closely match with the user input. The three methods are as follows. 


\subsection{Shape Template Matching}

In this method the user written character is scaled down to the dimension of the shape-template and each point on the user input is matched to the same position of the template. If there is a match then the probability of the character is increased. In this way the user input character is matched with the each template and the closest matched template will have the highest probability at the end of this recognition method.

\subsection{Pixel Density Matching}

The density of pixels varies for each character at specific position. So this data can be used in recognition characters as the density varies for each character. The density of pixel is matched from the templates to the user input at the same position of the character. If the densities are approximately equal then the probability for the character increases. In this way the pixel densities are matched for each template, therefore increasing the probability for the right character.

\subsection{Stroke Movement Matching}

This method uses the stroke template to match the movement of the strokes the templates with that of the user input. Each point of the user input is matched to the corresponding position in the template and if there is a match then the probability is increased for the specific character. In this method, the matching involves the inspection of the starting and ending positions of the character. Each character is written in different movements with respect to the initial position or starting point of the character. So this method efficiently recognizes the right character. Just like the previous method, the user input is matched with each template and the probability for the right character increases. By combining the above three methods and their probabilities, the right character is recognized.

\subsection{DETAILED ARCHITECTURE DESIGN}

Input from the Tablet \& Digitization: The process of Digitization happens at the run time itself. As the user inputs the data from the tablet, events are generated .These events are captured at the run time and a matrix of the dimension of the screen is used to initialize or used to store the positions where the pixels are plotted. The matrix minimizes the window where the pixels are drawn and captures the co-ordinates and initializes the corresponding positions of the matrix to 1 .

Pre-processing: This is an intermediate step between the input and the generation of training set. The input got from the user cannot be directly used to create a training set. There are few reasons because of which the input cannot be directly used. Firstly during the training process, the user is required to write one character at a time. The user may not write every character with the same dimension as the previous one. Hence this creates a problem which requires each character to be stored at variable dimension. Hence, the character written by the user must be scaled down or scaled-up according to the dimensions required by the training set. Hence the writing character must first be scaled then can be preceded to the generation of training set step. Secondly, the input is captured in the form of 2-D matrix. When this input matrix is scaled-up the pixel information stored in the matrix gets diluted, as the scaling up requires more number of pixels to be plotted. Hence some preprocessing must be done so that there are no gaps in the characters. Matrix formed by up-scaling the original matrix. If the input matrix is of small size than the training set dimension, then the input matrix must be scaleddown. This does not poses any problem as the pixels overlap. There is no gap in the characters matrix formed.

Generate Training Set: The training set is nothing but a file which contains the templates or the matrix generated from the pre-processing step. The format of the file can be of two forms. Firstly, the matrix can be stored as it is to the file with some delimiter characters to limit between each character. Secondly, only the coordinate values, where the pixels are plotted within a matrix can be stored along with the value initialized at the matrix. The training set file must be loaded into the recognition system before starting the process of recognition.

\section{Recognition Phase}

Input and Digitization: This step is same as the input and digitization of the training process, except for the fact that, there is some extra information's involved which must be stored at this step. In the recognition phase, the user can write only number of characters discretely. Hence to help in the segmentation of characters, the limits of each stroke of the pen is stored in some program variables. The limit of stroke across the $\mathrm{X}$-axis and across the Y-axis are stored at the runtime which can be used by the segmentation stage to segment each character and send to the recognition system. 
Divide lines: The first step after the input is captured, the matrix is to be divided the matrix into lines. Mainly, the limits of the lines are calculated at this step and stored in an array. The upper and lower limits are calculated. The main logic behind dividing lines in that, in a line of text there can be no row with empty pixel running from left to the rightmost position of the screen. Thin fact can be used to know when a line is started or stopped. Each row in the matrix is scanned to find out if the row is empty. If any value is initialized before the end of the row then it means that a lines upper limit is found. If an upper limit is found then the scanning of row continues until an empty row is encountered. An empty row in the matrix with only ' 0 ' initialized represents that the previous line has ended. And the next line is about to begin. So the upper and lower limits are stored and the process continues to find the next lines. After the lines are divided, the limits are sent for future steps which involve segmentation and recognition.

Segmentation: this is the next step after the lines are divided and limits calculated segmentation involves finding the character after combining the strokes. Each character can be written with one or a max of 3 strokes. It has been found that the strokes within a single character overlap most of the time. This fact is used in the segmentation .if two or more strokes overlap within the boundaries of each other than these strokes are combined together to form a single character .the boundaries are nothing but the $\mathrm{x}$ and $\mathrm{y}$ coordinate limits of each stroke which is stored in a matrix. After combining strokes to, the single character formed is stored in a separate matrix and is sent to the recognition phase. After it is recognized the next character is segmented and the process continues.

Recognition: Three steps are involved in the recognition of a character.

Shape Template Matching: Refer section 4.1

Pixel Density Matching: Refer section 4.2

Stroke Movement Matching: Refer section 4.3

Storing and displaying of output: after the character is recognized, the character is stored in a file or displayed on the screen as any specific area set aside for displaying the result. The result can be stored on a notepad file and saved. And the detailed architecture diagram is as follows in figure 2.

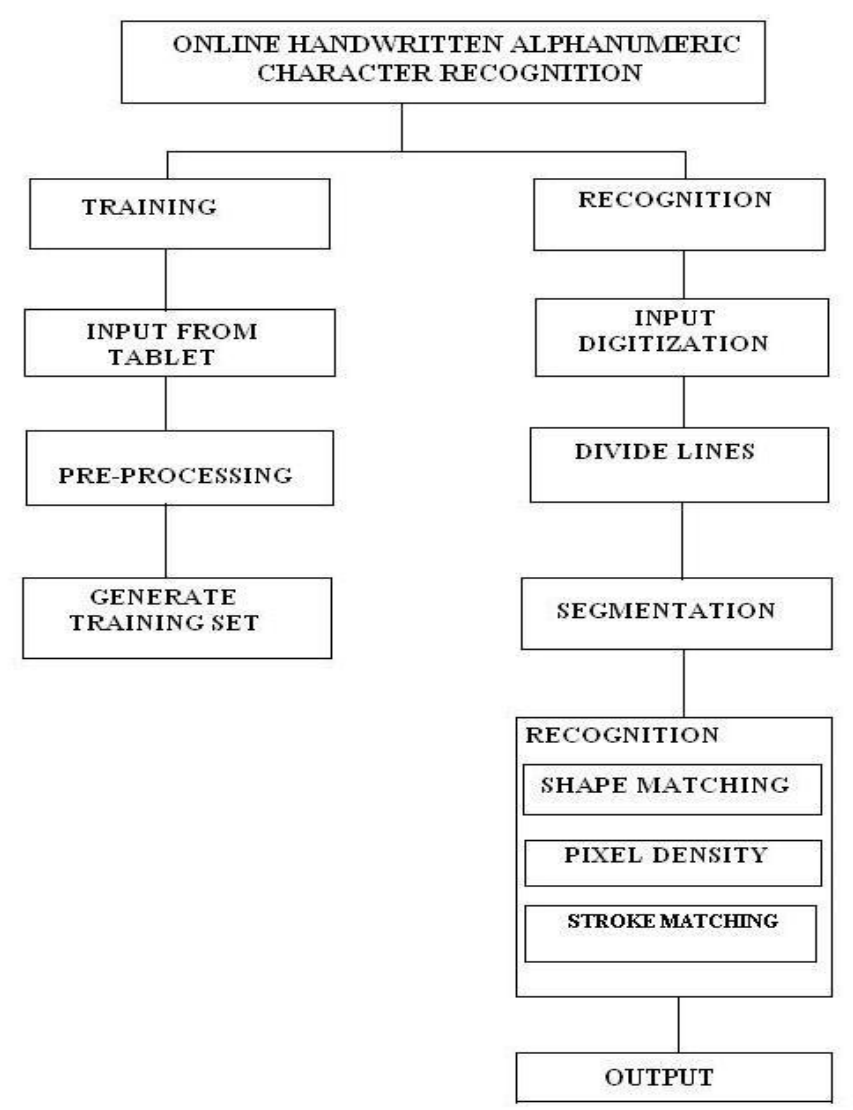

Fig 2: Detailed architecture 


\section{Flow Charts for Recognition and Training}

The below diagram depicts the flow of system in two different modes i.e., recognition and training mode. In the both the cases, the input is received from an external device like tablet which is given by the user. The user decides whether in which state the system should be in.

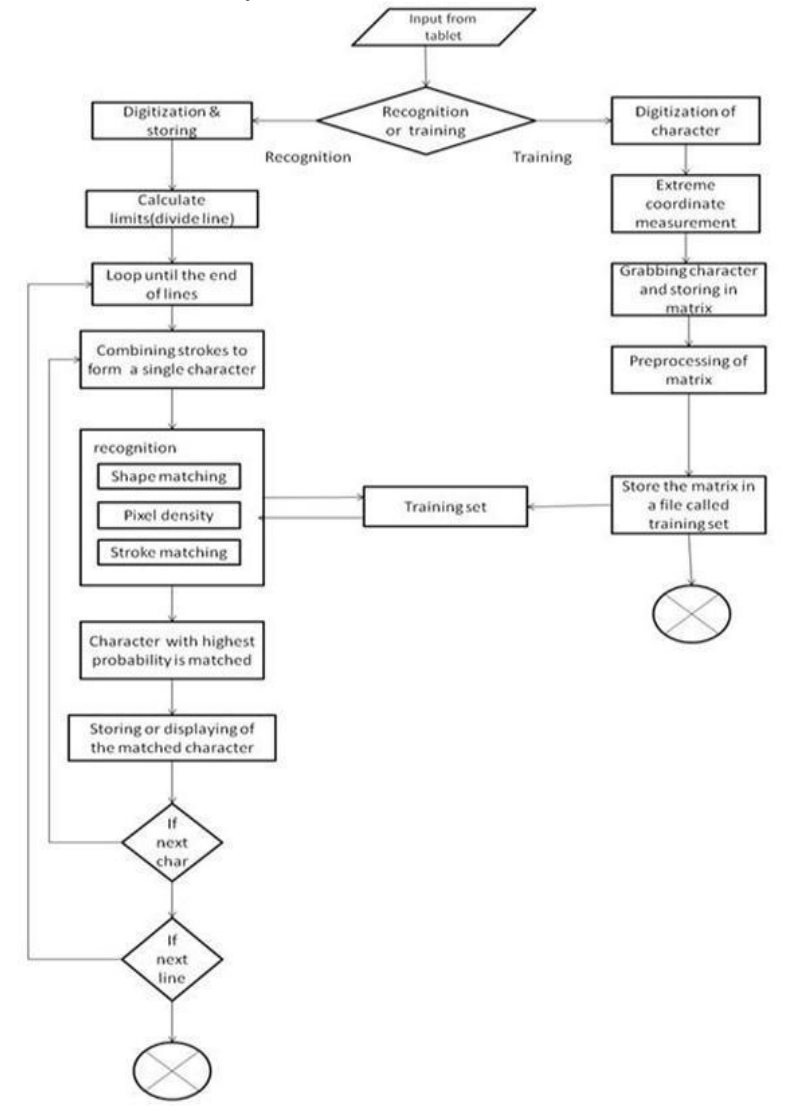

Fig 3: Flow chart of online hand written character recognition.

\section{Conclusion And Future Enhancement}

The main objective is to recognize online handwritten documents, which includes characters, words, lines, paragraphs etc. There is extensive work in the field of handwriting recognition, and a number of reviews exits.

In this approach, it is required to recognize handwriting by using templates. Along with this, it maintains unique user accounts, which enable a particular user to create his/her training sets. Also, online handwritten characters are recognized by a sequence of dominant points in strokes and sequence of writing direction between consecutive dominants points. The direction information of dominant points is used for character pre-classification and fine classification.

Recognition experiment have been conducted by using 62 character classes $(0-9, A-Z, a-z)$ of different styles. A remarkable aspect of the proposed approach is that, it is easily extensible to different character sets and different writing styles.

The system can be used in areas or fields where the data entry operations are required like manual handwritten documents to digitization of documents. The technique can be used for advanced message texting in mobile applications. The system can be enhanced to provide the output simultaneously as the user writes the data. Additional features like authentication issues, spell check algorithms, dictionary database, saving text in different fonts etc. In future, it is possible that, touch screens with this technique can become a solution for replacement of papers.

\section{References}

[1] Yoshida, K, 1982 C\&C System Research Laboratories Nippon Electric Co., Ltd

[2] Zafar, M.F. Informatics Complex, Islamabad Mohamad, D. ; Anwar, M.M. Multitopic Conference, 2006. INMIC '06. IEEE.

[3] Chiang, C.C. CompEuro '91. Advanced Computer Technology, Reliable Systems and Applications. 5th Annual European Computer Conference. Proceedings. 1991

[4] Liangwen Zhang Intelligent Computing and Intelligent Systems (ICIS), 2010 IEEE International Conference Nankai Univ., Tianjin, China. 
[5] Yongqiang Wang; Qiang Huo; Yu Shi; Frontiers in Handwriting Recognition (ICFHR), 2010 International Conference on Digital Object Identifier: 10.1109/ICFHR.2010.86 Publication Year: 2010

[6] Del Vescovo, G.; Rizzi, A.; Granular Computing, 2007. GRC 2007. IEEE International Conference on Digital Object Identifier: 10.1109/GrC.2007.141 Publication Year: 2007.

[7] Garain, U.; Chaudhuri, B.B.; Pal, T.T.; Pattern Recognition, 2002. Proceedings. $16^{\text {th }}$ International Conference on Volume: 3Digital Object Identifier: 10.1109/ICPR.2002.1047820 Publication Year: 2002

[8] Sundaram, Suresh; Ramakrishan, A. G.; Document Analysis and Recognition (ICDAR), 2011 International Conference on Digital Object Identifier: 10.1109/ICDAR.2011.237 Publication Year: 2011.

[9] Ma, Long-long; Liu, Hui-dan; Wu, Jian; Document Analysis and Recognition (ICDAR), 2011 International Conference on Digital Object Identifier: 10.1109/ICDAR.2011.50 Publication Year: 2011. 